\section{Outcome of cataract extraction and posterior chamber intraocular lens implantation following glaucoma filtration surgery}

'VST Centre for Glaucoma Care, LV Prasad Eye Institute,

Hyderabad, India

${ }^{2}$ International Centre for Advancement of Rural Eye Care, LV Prasad Eye Institute,

Hyderabad, India

Correspondence:

AK Mandal,

VST Centre for Glaucoma

Care,

LV Prasad Eye Institute,

LV Prasad Marg,

Banjara Hills,

Hyderabad 500 034,

AP, India

Tel: + 91040354 8267;

Fax: + 910403548271 .

E-mail: mandal@|vpei.org

Received: 15 October 2003 Accepted: 20 April 2004

Published online:

6 May 2005

Presented as a paper at the Annual meeting of the American Academy of Ophthalmology, Orlando, Florida, 20-23 October, 2002.

The authors have no proprietary interest in any of the materials or techniques used in this study
Abstract

Purpose To evaluate the outcome of cataract extraction (CE) after glaucoma filtering surgery (GFS).

Methods A total of 77 eyes (77 patients) who underwent $C E$ with posterior chamber intraocular lens (PCIOL) implantation following GFS by a single surgeon were reviewed. Main outcome measures were preoperative and postoperative intraocular pressures (IOPs), visual acuities, medications, astigmatism, bleb survival, time of surgical failure, complications, and success rate. Results Mean time interval between GFS and CE was $46.8 \pm 50.9$ months (range, 2-348 months). The mean preoperative IOP was $13.9 \pm 4.7 \mathrm{mmHg}$ (range $3-27 \mathrm{mmHg}$ ) and mean postoperative IOP at 3 weeks was $13.6 \pm 5.5 \mathrm{mmHg}$ (range, $6-44 \mathrm{mmHg}$ ). The mean follow-up was $19.5 \pm 20.1$ months (range, 1.4-73 months; median 10.6 months). Complete success was achieved in 59 eyes $(76.7 \%)$. The cumulative probability of complete success was $91.3 \pm 3.7,82.0 \pm 5.6$ and $78.1 \pm 6.5 \%$ at the end of 6 months, 1, and 2 years, respectively. Visual acuity before CE was $\leqslant 20 / 50$ in all eyes $(100 \%)$. Visual acuity at last visit was $\geqslant 20 / 40$ in 33 eyes $(42.8 \%), 20 / 50-20 / 80$ in 30 eyes $(39.0 \%), \leqslant 20 / 100$ in 14 eyes $(18.2 \%)$. Risk factors identified for qualified success included age at $C E>60$ years, interval of $\leqslant 5$ months between GFS and CE, use of preoperative glaucoma medications, and postoperative IOP $>19 \mathrm{mmHg}$ within 2 weeks. Conclusions IOP and bleb function was maintained after CE with PCIOL implantation following successful GFS with good visual recovery.
AK Mandal', V Chelerkar', SS Jain' and R Nutheti

Eye (2005) 19, 1000-1008. doi:10.1038/sj.eye.6701703; published online 6 May 2005

Keywords: cataract extraction; PCIOL, glaucoma filtering surgery

Introduction

Glaucoma and cataract often coexist in the same patient. Although these are two different entities with an independent natural course, they are inter-related when they have to be managed together. This is important because management of one condition may affect the clinical course of the other. Use of glaucoma medications or surgery is known to accelerate cataract formation, ${ }_{1}^{1-3}$ while surgery for cataract may disturb glaucoma control. The aim is to provide the best possible visual acuity and good glaucoma control. Depending on the severity of glaucoma and cataract, there exist three options for management of coexisting cataract and glaucoma. These include cataract surgery alone with medical control of glaucoma, glaucoma filtration surgery (GFS) followed by cataract extraction (CE) (when necessary), or combined cataract and glaucoma surgery. ${ }^{4,5}$ The advantages of GFS first followed by $C E$ include improved intraocular pressure (IOP) control, and the potential for visual improvement even before $\mathrm{CE}$ (in patients on miotics). However, the disadvantages include increased cost of two procedures, progression of cataract after GFS and jeopardized function of the bleb after CE. The aim of the present study was to evaluate the outcome of cataract surgery after GFS and filtering bleb function following $\mathrm{CE}$. 


\section{Methods}

We identified the eligible patients from the computerized surgical log of the patients that underwent CE following a GFS between October 1993 and December 1999 at LV Prasad Eye Institute (LVPEI), Hyderabad, India. All the cataract surgeries were performed by a single surgeon (AKM). Prior approval for study was sought from the Ethics committee of LVPEI before reviewing the medical records. We identified 83 patients (103 eyes) for the study. Demographic data reviewed included age at the time of $\mathrm{CE}$, gender, the type of glaucoma, best-corrected visual acuity, IOP, type, and number of antiglaucoma medications preoperatively, the appearance of filtering bleb (pre- and post-CE), time interval between trabeculectomy and $\mathrm{CE}$, the length of cataract incision in clock hours, location of cataract incision (clear corneal, temporal limbal), number of sutures required for closure of the wound, type of intraocular lens (IOL) placement (endocapsular $v$ s sulcus fixated), intraoperative and postoperative complications and number of postoperative medications. The IOP, bleb appearance, and visual acuity at 1 day, 1, 3, and 6 weeks, and every 3 months thereafter were assessed. The amount of astigmatism after CE was also assessed.

A total of 91 eyes $(94.8 \%)$ had undergone a trabeculectomy, four eyes $(4.2 \%)$ had iridencleisis and one eye $(1.0 \%)$ underwent Scheie's procedure for control of glaucoma. All the glaucoma surgeries were performed in the superior quadrant of the eye without use of any antifibrotic agent. All patients underwent a standard extracapsular CE (ECCE) with posterior chamber IOL (PCIOL) implantation, the cataract incision being made in the clear cornea in front of the bleb, avoiding trauma to the bleb, or in the temporal limbus. Anterior capsulotomy was carried out with the envelope technique, pressure-counter pressure was applied to deliver the nucleus and the cortical remnants were aspirated using the classical Simcoe cannula. A polymethylmethacrylate lens was placed in the capsular bag, and the wound closed with 10-0 nylon interrupted sutures. In those eyes that had a small pupil during cataract surgery, sphincterotomy and pupilloplasty were performed to facilitate easy removal of the nucleus. Postoperatively, all patients were treated with betamethasone $0.1 \%$ eye drops in tapering doses for 6 weeks, cyclopentolate 1\% eye drops for 4 weeks and gentamicin $0.3 \%$ eyedrops for 1 week. Glaucoma medications were tailored based on the disc damage, visual field loss, and level of IOP following CE. All patients were refracted at the end of 6 weeks and if astigmatism was $>4.00 \mathrm{D}$, suture removal was performed and refraction was repeated a week later.
The outcome measures included pre- and postoperative IOP, visual acuity, glaucoma medications, astigmatism, and bleb function. We defined complete success as IOP $\leqslant 21 \mathrm{mmHg}$ without any medications after 2 months of CE and no bleb revision or further glaucoma surgery undertaken for long-term control of IOP. Qualified success was defined as IOP $\leqslant 21 \mathrm{mmHg}$ with medications 2 months after CE for IOP control. Failure was defined as when further glaucoma surgery was required for IOP control. We defined improvement in vision as a difference in acuity from the baseline by $\geqslant 2$ Snellen lines and considered the vision to have worsened if the acuity dropped by $\geqslant 2$ Snellen lines from the baseline.

For purposes of statistical analysis, one randomly chosen eye of each patient was considered. Results are expressed as mean \pm standard deviation. Each variable was evaluated for association with trabeculectomy survival using Kaplan-Meier survival analysis. Cox multivariate regression analysis using forward stepwise method was used to determine the risk factors that were independent predictors of qualified success or failure. All the pre- and postoperative factors were considered as variables into this model. Differences in pre- and post-CE IOP were examined for statistical significance using paired ' $t$ '-test and independent sample ' $t$ '-test was used to compare the mean IOPs and duration of follow-up between the complete success and qualified success groups. A $P$-value of $<0.05$ was considered statistically significant.

\section{Results}

We identified 103 eyes of 83 consecutive patients who underwent GFS followed by CE. We excluded six patients because of inadequate follow-up ( $<6$ weeks) resulting in a study population of 96 eyes of 77 patients. The mean age at the time of CE was $61.7 \pm 10.6$ years (range, 29-85). Demographic data and characteristics of the patients are listed in Table 1 .

The mean interval from glaucoma surgery to $\mathrm{CE}$ was $46.8 \pm 50.8$ months (median 32.4; range 2-348). All patients underwent standard ECCE with PC IOL implantation; the incision was clear corneal in 70 eyes $(90.9 \%)$, and limbal in seven eyes $(9.1 \%)$. The corneal incision was superiorly located just in front of the bleb in these eyes (Figures 1-4). The placement of the IOL was endocapsular in 93 eyes $(96.8 \%)$, two eyes $(2.1 \%)$ had a sulcus fixated PC IOL, and one eye (1.0\%) was left aphakic because of the absence of capsular support as the lens was subluxated and removed in toto.

Of the 77 eyes, 24 (31.2\%) required the use of glaucoma medications prior to CE with $17(70.8 \%)$ of them requiring single medication and seven $(29.2 \%)$ requiring 
Table 1 Patient demographics (96 eyes of 77 patients with CE after GFS)

\begin{tabular}{lc}
\hline Demographics & No. $(\%)$ \\
\hline $\begin{array}{l}\text { Age (years) } \\
\text { Mean }\end{array}$ & $61.7 \pm 10.6$ \\
Range & $29-85$ \\
Race & \\
White & \\
Black & 0 \\
Asian & 0 \\
& $109(100)$ \\
Gender & \\
Male & \\
Female & $52(67.1)$ \\
& $25(32.9)$ \\
Type of glaucoma & \\
POAG & \\
PACG & $43(55.8)$ \\
Pseudoexfoliative glaucoma & $28(36.4)$ \\
Uveitic glaucoma & $3(3.9)$ \\
Steroid-induced glaucoma & $2(2.6)$ \\
\end{tabular}

POAG = primary open angle glaucoma; PACG = primary angle closure glaucoma.

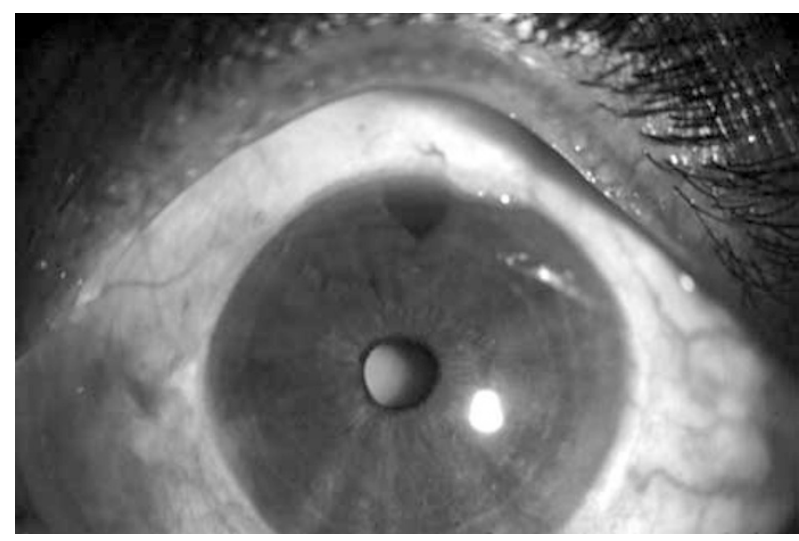

Figure 1 Clinical photograph of an eye with cataract 3 years following GFS.

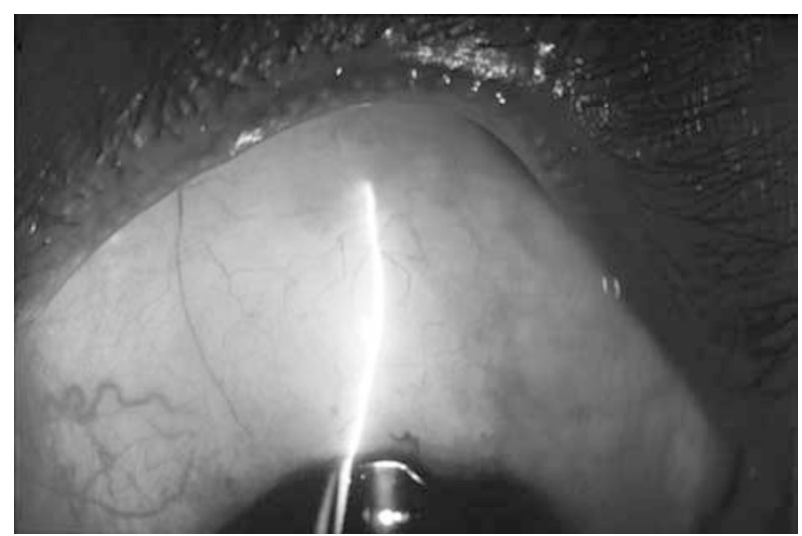

Figure 2 Bleb appearance of the same eye as shown in Figure 1.

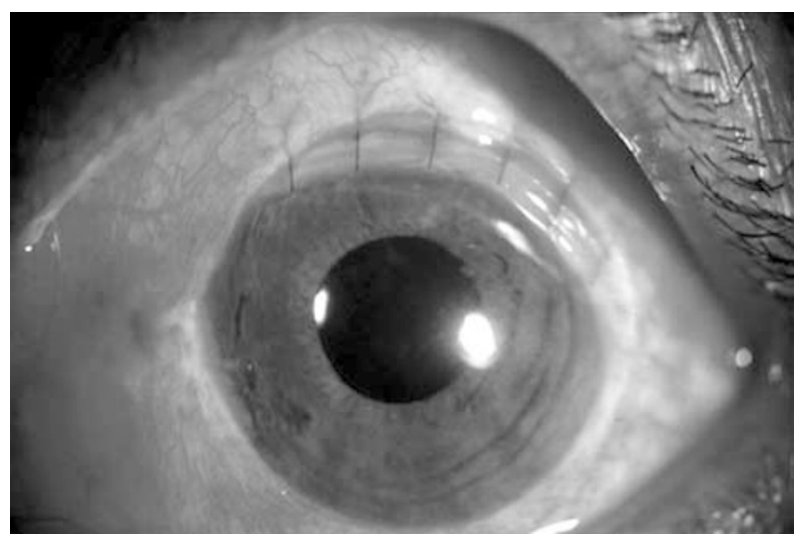

Figure 3 Clinical photograph of the eye in Figure 1 after CE on the first postoperative day.

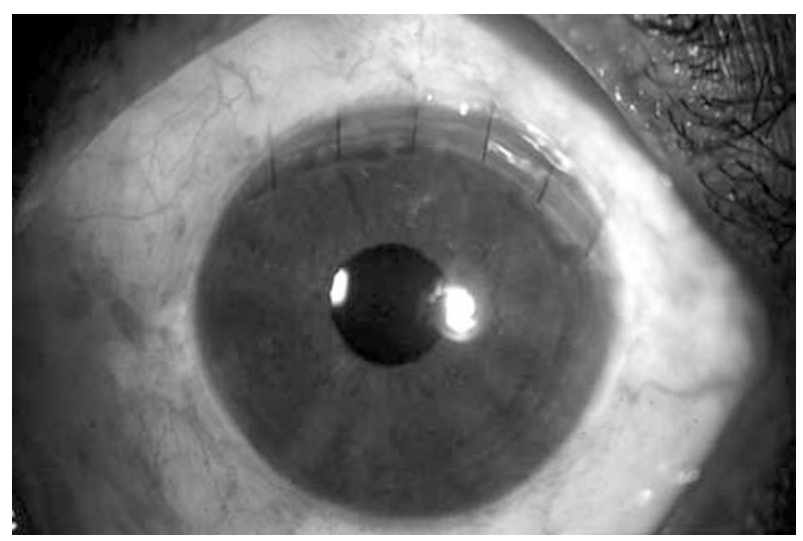

Figure 4 Clinical photograph of the eye seen in Figure 3 three months after CE.

two medications. Of these 24 eyes, 14 (58.3\%) needed to continue with one medication after CE.

Complete success as defined in the present study was achieved in 59 eyes (76.6\%), qualified success in 17 eyes $(22.1 \%)$, and only one eye $(1.3 \%)$ was a failure. The cumulative probability of complete success was $91.3 \pm 3.7,82.0 \pm 5.6$, and $78.1 \pm 6.5 \%$ at 6 months, 1 , and 2 years, respectively (Figure 5 ). The mean follow-up was $19.5 \pm 20.1$ months (range, 1.4-73 months; median 10.6 months). The eyes in complete success group had a mean follow-up of $14.6 \pm 17.1$ months (range, 1.4-61 months, median 6.9 months) while those in the qualified success group had a mean follow-up of $35.3 \pm 21.7$ months (range, 2-73 months, median, 37.3 months). This difference in follow-up between the groups was statistically significant $(P<0.0001)$.

The mean pre-CE IOP was $13.9 \pm 4.7 \mathrm{mmHg}$ (range 3-27 mmHg). The pre-CE IOP of eyes that achieved complete success was $13.7 \pm 4.7 \mathrm{mmHg}$ (range, 3-27) $(n=72)$ and of those that were qualified success or failure 
was $14.3 \pm 4.5 \mathrm{mmHg}$ (range, 4.0-23.0). This difference in IOP between the groups was not statistically significant $(P=0.64)$. The trends in IOP using a box plot at various time points following $\mathrm{CE}$ in the complete success group is depicted in Figure 6. The mean maximum IOP during the first 2 weeks post-CE was $13.6 \pm 5.5 \mathrm{mmHg}$ (range, 6-44). During this period IOP $>25 \mathrm{mmHg}$ was noted in three eyes and these eyes required single glaucoma medication for control of IOP. Although postoperative

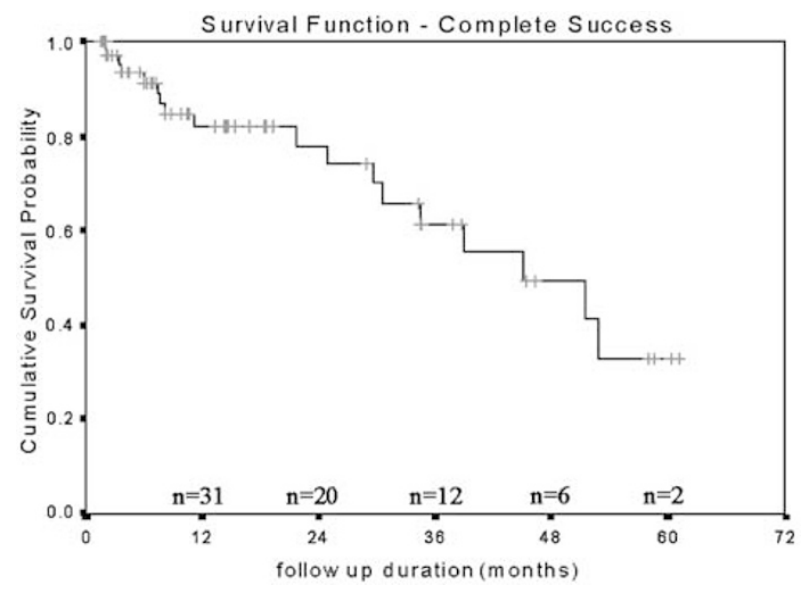

Figure 5 Kaplan-Meier survival curve for IOP control in eyes undergoing CE after GFS.

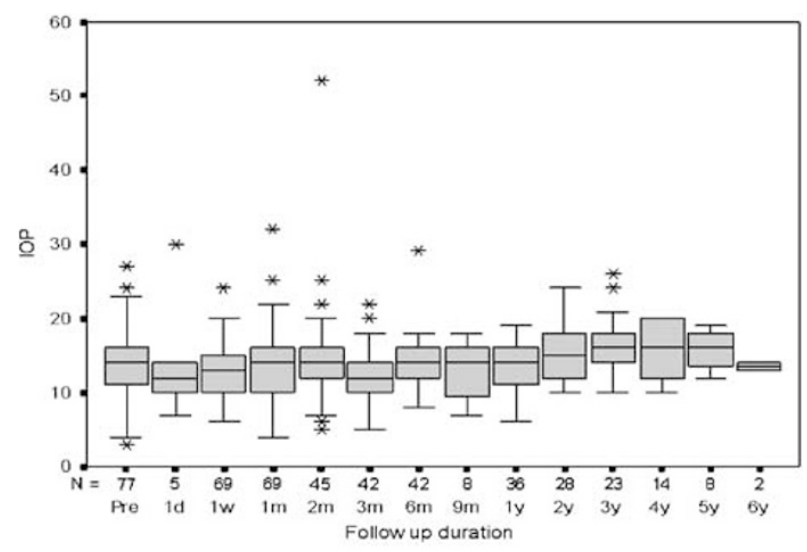

Figure 6 Box plot of IOP changes at various time points in the complete success group.
IOP as a continuous variable was not associated with trabeculectomy survival, postoperative IOP $>19 \mathrm{mmHg}$ in the early period ( $<2$ weeks) was significantly associated with qualified success $(P=0.014)$ (Table 2$)$ and this association remained significant (risk ratio, 11.06; $95 \%$ CI 1.8, 67.0, $P=0.020$ ) after multivariate analysis (Table 3).

Other preoperative variables associated with qualified success by univariate (Kaplan-Meier) analysis included

Table 2 Factors associated with qualified success in eyes having CE after trabeculectomy

\begin{tabular}{llll}
\hline Factor & $\mathrm{N}$ & $\begin{array}{c}\text { Survival } \\
(\%)^{\mathrm{a}}\end{array}$ & $\mathrm{P}^{*}$ \\
\hline $\begin{array}{l}\text { Age at CE (years) } \\
\quad \leq 60\end{array}$ & 35 & $48 \pm 4$ & \\
$\quad>60$ & 42 & $33 \pm 5$ & 0.047
\end{tabular}

Interval between GFS and CE (months)

$\begin{array}{rrrr}\leq 5 & 4 & 5 \pm 1 & \\ >5 & 73 & 41 \pm 4 & 0.049\end{array}$

Early postoperative maximum IOP $(<2$ weeks) (monthly)

$\begin{array}{lrr}\leq 19 \mathrm{mmHg} & 67 & 42 \pm 4 \\ >19 \mathrm{mmHg} & 6 & 10 \pm 3\end{array}$
0.014

Pre-CE IOP (monthly) $\leq 15 \mathrm{mmHg}$

$>15 \mathrm{mmHg}$

$\begin{array}{ll}48 & 45 \pm 4 \\ 29 & 34 \pm 7\end{array}$

Type of glaucoma

POAG

PACG

$43 \quad 38 \pm 4$

$28 \quad 47 \pm 6$

0.574

Use of antiglaucoma medications No

Yes

$24 \quad 25 \pm 7 \quad 0.001$

Intraoperative complications/ manipulations

\begin{tabular}{lrrr} 
None & 70 & $38 \pm 7$ & \\
Any & 7 & $47 \pm 6$ & 0.571 \\
\hline
\end{tabular}

GFS = glaucoma filtering surgery; $\mathrm{CE}=$ cataract extraction; $\mathrm{POAG}=$ primary open angle glaucoma; PACG = primary angle closure glaucoma. ${ }^{a}$ Kaplan-Meier survival analysis estimate of 12 -month survival rate. ${ }^{*}$ Log-rank $P$-value.

Table 3 Cox multivariate regression analysis of pre- and postoperative factors

\begin{tabular}{lrr}
\hline Factor & Risk ratio $(95 \%$ CI $)$ & $\mathrm{P}$ \\
\hline Age at CE: $>60$ years & $3.47(1.1,11.3)$ & 0.039 \\
Maximum postoperative IOP within 2 weeks: $>19 \mathrm{mmHg}$ & $11.06(1.83,67.0)$ & 0.009 \\
Interval between GFS and CE: $\leqslant 5$ months & $22.42(1.62,310.9)$ & 0.020 \\
Use of anti-glaucoma medications: Yes & $6.31(1.95,20.49)$ & 0.002 \\
\hline
\end{tabular}


age $>60$ years $(P=0.047)$, time interval of $\leq 5$ months between GFS and CE $(P=0.049)$, and pre-CE use of glaucoma medications (vs none, $P=0.001$ ) (Table 2). After multivariate analysis too, age $>60$ years, early postoperative IOP within 2 weeks of CE of $>19 \mathrm{mmHg}$, time interval between GFS and CE of $\leqslant 5$ months and pre-CE use of glaucoma medications remained significant (Table 3). However, none of the other factors such as intraoperative iris manipulations $(P=0.8358)$, early postoperative anterior chamber inflammation $(P=0.5388)$, late endophthalmitis $(P=0.7189)$, incision site, or the length of the incision were associated with qualified success.

Three eyes of two patients with uveitic glaucoma had good IOP control and bleb function. The glaucoma was under control prior to $\mathrm{CE}$ and the eyes were free from inflammation/uveitic activity for more than 6 months before CE. There was no complication or difference between these and other patients. Of these three eyes, two $(66.7 \%)$ eyes achieved qualified success and one $(33.3 \%)$ eye was a complete success. The patient with steroid-induced glaucoma maintained good IOP control without any additional medication.

Intraoperative complications occurred in two eyes $(2.6 \%)$, intraoperative iris manipulations in five eyes $(6.5 \%)$, and postoperative complications developed in 10 eyes $(13 \%)$. The intraoperative complications included posterior capsular tear with (one) and without vitreous loss (one) and the intraoperative iris manipulation was required in eyes with small nondilating pupil in the form of pupilloplasty. Postoperative complications included mild-to-moderate anterior chamber reaction (six eyes) that resolved completely with anti-inflammatory treatment, corneal oedema (two eyes), iris prolapse in the immediate postoperative period (one eye), encapsulated bleb (one eye), aqueous misdirection (one eye), late onset endogenous endophthalmitis (two eyes), and overhanging bleb (one eye). Both the eyes that developed endophthalmitis (24.4 and 46 months post-CE in the right and left eye, respectively) underwent pars plana vitrectomy with intraocular antibiotic injection and the final visual acuity was 20/40 and 20/100 in the right and left eye, respectively. The IOP was under control without medication in right eye but the left eye required one medication. No risk factors could be identified in this patient. The eye that developed an overhanging bleb (encroaching superior $1 / 3$ of cornea) underwent bleb revision in the form of excision of the overhanging bleb and resuturing of the conjunctiva over the site of trabeculectomy. The final bleb was functional and IOP remained under control without medication. One eye had a failed filtration function 26 months after the CE that required second glaucoma surgery.
Hypotony (IOP $\leqslant 6 \mathrm{mmHg}$ ) before CE was noted in five eyes $(6.5 \%)$ and transient hypotony after CE was seen in 12 eyes (15.6\%). All eyes except one recovered from hypotony at the last follow-up and none of the eyes demonstrated hypotony maculopathy. Of these 17 eyes, seven $(41.2 \%)$ eyes attained a visual acuity of $\geqslant 20 / 50$.

Types of bleb appearance pre- and post-CE have been compared in Table 4. A functional bleb was seen in 75 patients $(97.4 \%)$ prior to CE. However, a bleb was seen in 72 patients $(93.5 \%)$ after CE (Figures 7 and 8 ). This difference was statistically not significant $(P=0.4391)$.

Table 4 Comparison of bleb appearance before and after CE in 77 patients

\begin{tabular}{lcrc}
\hline Type of bleb & $\begin{array}{l}\text { Pre-CE } \\
\text { no. (\%) }\end{array}$ & $\begin{array}{r}\text { Post-CE } \\
\text { no. }(\%)\end{array}$ & P \\
\hline Diffuse & $46(61.3)$ & $46(63.9)$ & 0.7489 \\
Low & $15(20.0)$ & $20(27.8)$ & 0.2684 \\
Cystic & $14(18.7)$ & $6(83.0)$ & 0.0677 \\
\hline
\end{tabular}

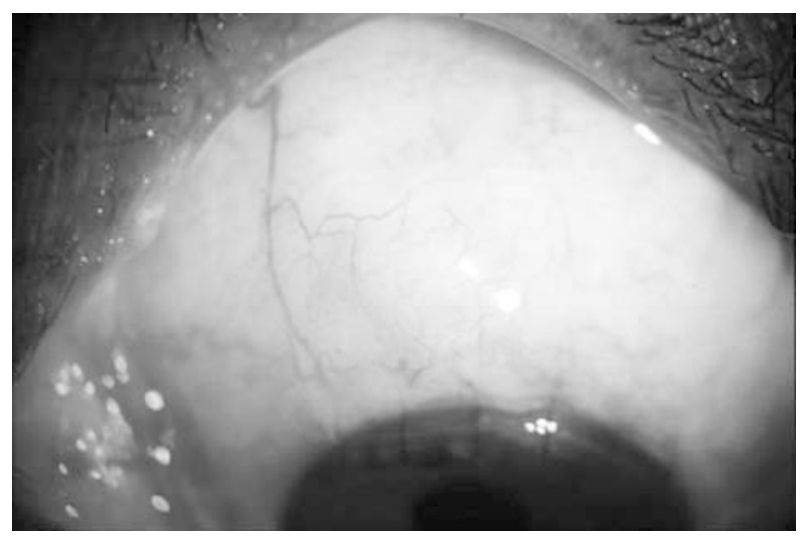

Figure 7 Bleb appearance of the eye seen in Figure 1 one year after $\mathrm{CE}$.

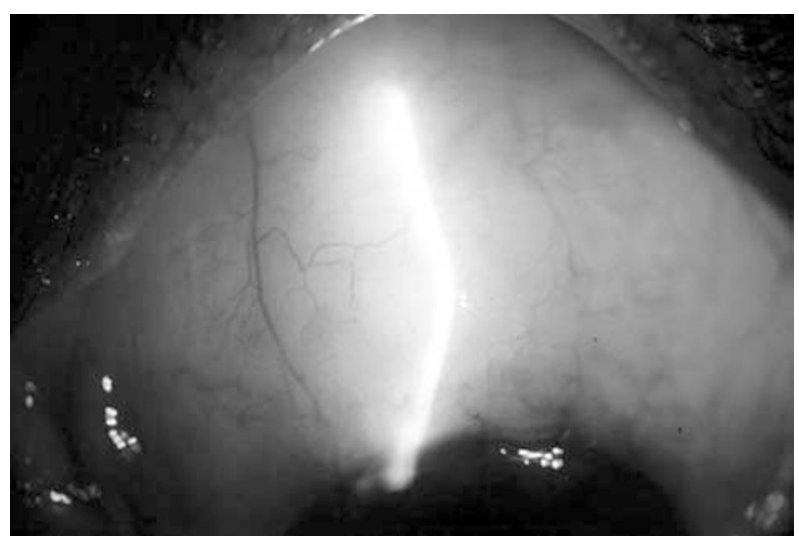

Figure 8 Bleb appearance in slit-illumination of the eye shown in Figure 7. 


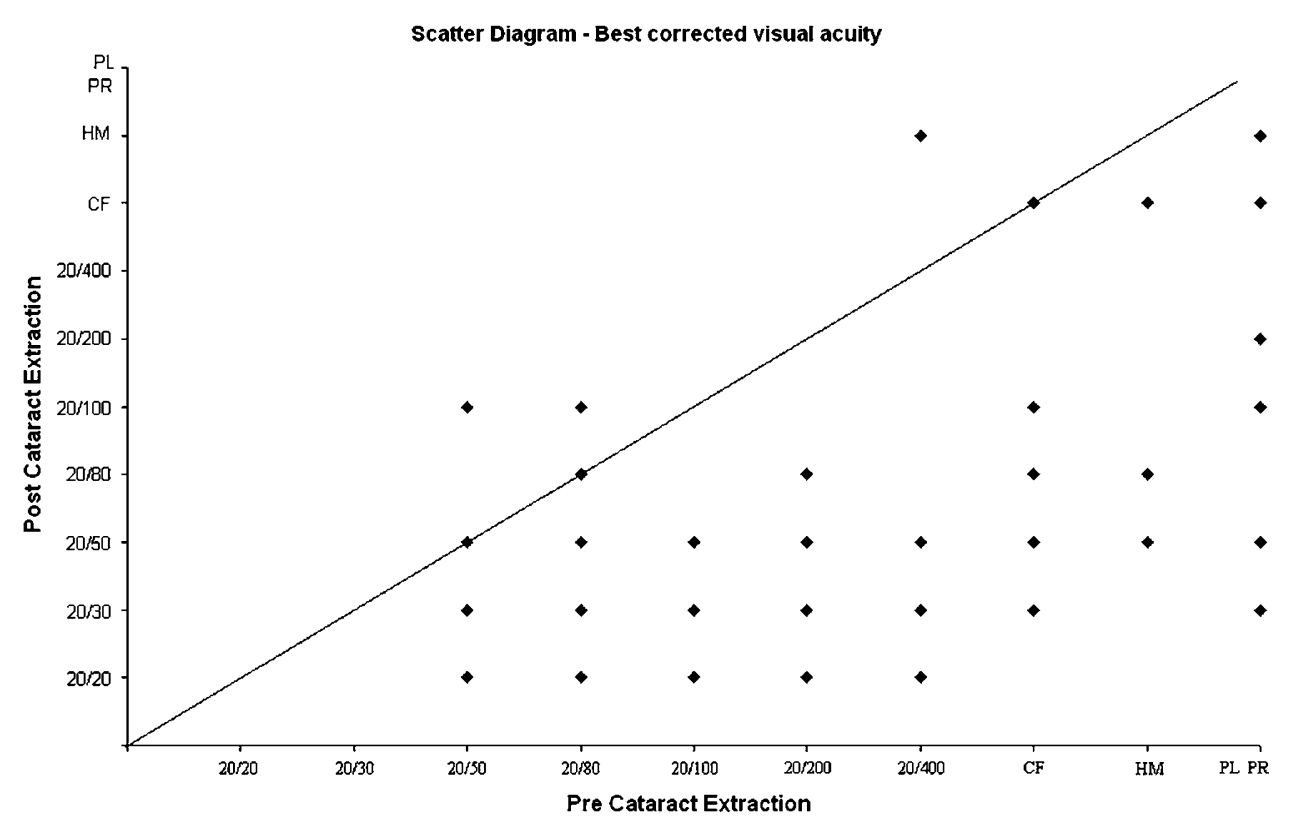

Figure 9 Scatter plot of pre-CE vs post-CE visual acuity.

Improvement in vision as defined in the present study was found in 63 eyes (81.8\%), while 12 eyes (15.6\%) retained the baseline acuity. Worsening of vision was found in two eyes (2.6\%) only (Figure 9). All the eyes $(n=77)$ had $\leqslant 20 / 50$ before CE and at the last visit following CE a visual acuity of $\geqslant 20 / 40$ was noted in 33 eyes $(42.8 \%), 20 / 50$ to $20 / 80$ in 30 eyes $(39.0 \%)$, and $\leqslant 20 / 100$ in 14 eyes $(18.2 \%)$.

At 6 months postoperatively, astigmatism of $\leqslant 2.0 \mathrm{D}$ was noted in 44 eyes $(57.1 \%)$ and $>2.0 \mathrm{D}$ in 33 eyes $(42.9 \%)$. Of the 33 eyes with astigmatism $>2 \mathrm{D}$, two eyes $(6.1 \%)$ had astigmatism $>8.0 \mathrm{D}$ that reduced to $3 \mathrm{D}$ after suture removal in one eye and one eye underwent astigmatic keratotomy.

\section{Discussion}

The management of coexisting cataract and glaucoma poses a dilemma to most glaucoma surgeons. While medical treatment alone may not often suffice, surgical management is often difficult. The surgical management of coexisting cataract and glaucoma remains controversial.

Performing a cataract surgery after a successful trabeculectomy frequently triggers bleb failure. ${ }^{4,6,7}$ Previous investigators have looked at the effect of $\mathrm{CE}$ on IOP in patients with pre-existing filtering blebs. ${ }^{2,8-11}$ Caprioli et al ${ }^{9}$ found no difference in IOP control over time in a matched case-control study comparing patients with existing blebs (40 eyes) with those with existing blebs undergoing clear cornea phacoemulsification
CE (PECE) (40 eyes). On the contrary, Chen et $a l^{2}$ reported on 115 eyes in a retrospective review of patients with functioning filtering blebs in whom either ECCE (58 eyes) or PECE (57 eyes) was performed using both clear corneal and scleral approaches. In their study, for the group as a whole, $30.4 \%$ required either additional medication or bleb needling to maintain IOP control at a mean follow-up of 21 months. Additional 11 eyes (9.6\%) required glaucoma surgery. A Cox proportional hazards model found the relative hazard of requiring glaucoma surgery was 3.0 (95\% confidence interval, $0.7,12.8)$ for those undergoing ECCE as compared with PECE. Manoj et $a l^{10}$ compared ECCE with PECE in patients with preexisting filtering blebs. IOP increased significantly (mean increase, $1.9 \mathrm{mmHg}$ ) in the ECCE group (34 eyes), but remained unchanged in the PECE group (21 eyes).

Medication requirements were also greater in the ECCE group. ${ }^{10}$ Gunning and Greve ${ }^{8}$ reported a mean decrease of $1.5 \mathrm{mmHg}$ (not statistically significant) in individuals undergoing ECCE with pre-existing filters. Finally, Burratto and Ferrari ${ }^{11}$ studied individuals undergoing either ECCE or PECE with pre-existing blebs. A recently published evidence-based update mentioned that the existing data in the literature regarding the effect of $\mathrm{CE}$ on bleb function have been inconclusive. ${ }^{12}$

In the present series, considerable fluctuations in IOP occurred during the first few postoperative weeks and required additional medical therapy. The IOP control tended to stabilize after the second postoperative month. An important observation in the present study was that some patients had a rise in IOP in the postoperative 
period despite the presence of a functioning filtering bleb. The possible causes of a rise in IOP usually include inflammatory cells/debris in the angle of the anterior chamber or outflow channels, surgical hyphaema, residual lens cortical matter, and retained viscoelastic material. ${ }^{4}$ Most of the pressure spikes are transient and can be controlled with antiglaucoma medications as seen in our series. Most patients had stable IOP control after the second postoperative month.

Following CE with PCIOL implantation in an eye with a functional filtering bleb, the early postoperative IOP may rise to high levels in the presence of trabeculectomy. Several reports have indicated changes in mean IOP after CE ranging from a decrease of $0.8 \mathrm{mmHg}$ to increase of $6.6 \mathrm{mmHg}$ after a mean follow-up ranging from 8 to 70 months. ${ }^{5,11,13-19}$ We found a mean maximum IOP within 2 weeks of $\mathrm{CE}$ was $13.6 \mathrm{mmHg}$. A marked postoperative IOP rise is commonly encountered after uncomplicated ECCE in glaucoma patients. ${ }^{20}$ In the present study, eyes with postoperative IOP spike greater than $19 \mathrm{mmHg}$ were 11 times more likely to be associated with qualified success (risk ratio, 11.06; 95\% CI 1.83-67.0). These eyes need careful monitoring and additional glaucoma medications may be useful to control this transient rise in IOP with the occasional need for a repeat filtering surgery. The risk factors affecting the survival of a bleb are aphakia, darker race, inflammation, or neovascularization of the anterior chamber structures, and additional trauma. ${ }^{21}$ Additionally, the incision of a cataract surgery acts as an insult to the normal functioning of the bleb. Mechanisms of bleb failure following additional intraocular surgery include inflammation of the conjunctiva adjacent to the filtering bleb, and transient decrease in aqueous humour production in the first few years after cataract surgery., ${ }^{4,22}$ Increased postoperative intraocular inflammation accelerates late bleb scarring. Postoperative shallow anterior chamber can result in peripheral anterior synechiae or synechiae formation around the internal ostium. ${ }^{7}$ The bleb function and IOP stabilize between 2 and 6 months postoperatively.

Bigger and Becker ${ }^{22}$ studied the effect of uncomplicated cataract surgery on glaucoma control. They postulate that a decrease in aqueous humour production in the postoperative period results in a decreased flow through the sclerostomy. Consequently, the blebs flatten, increasing contact between conjunctiva and episcleral tissue leading to scarring of the bleb. The interval between filtering surgery and CE may be an important variable. Longer interval between the two surgeries has fewer adverse effects on the pressure control. Mature filtration blebs are probably more resistant to insult of additional intraocular surgery. Most filtering bleb failures occur in the first year after trabeculectomy. Among the shorter interval group, some blebs would have failed even without further cataract surgery. It is best to allow the filtering bleb to settle down for a longer period of time before planning for cataract surgery.

Adding a cataract operation to a planned trabeculectomy appears to diminish the IOP-lowering effect of trabeculectomy by approximately $2-4 \mathrm{mmHg}$ on average. The implication is that when IOP is of paramount importance, it may be better to separate the cataract and trabeculectomy procedures. ${ }^{12}$

Phacoemulsification appears to have fewer adverse effects on the postoperative IOP control than a standard ECCE. ${ }^{7}$ Advantages of phacoemulsification over ECCE include a smaller incision, more controlled anterior chamber depth during the intra- and postoperative period and fewer intraoperative manipulations resulting in better IOP control. In the present series, five patients underwent concomitant pupil surgery like synechiolysis, pupilloplasty, and surgical iridoplasty. This was found to be associated with increased postoperative inflammation in the early phase. Appropriate treatment of inflammation and increased IOP in the early postoperative period is mandatory. Intraoperative complications are another risk factor for bleb failure and should be avoided or reduced.

Chen $e t a l^{2}$ found the risk factors for worsening of IOP control as age $\leqslant 50$ years, preoperative IOP $\geqslant 10 \mathrm{mmHg}$, intraoperative iris manipulation, and early postoperative $\mathrm{IOP} \geqslant 25 \mathrm{mmHg}$. We identified several preoperative variables that were significantly associated (by univariate Kaplan-Meier survival analysis) with loss of IOP control, including age at $\mathrm{CE}$ of $>60$ years, time interval $\leqslant 5$ months between trabeculectomy and $\mathrm{CE}$, early postoperative maximum IOP (within 2 weeks) of $>19 \mathrm{mmHg}$. All these factors were found to be significant with qualified success even in the Cox multivariate analysis (Table 3). Chen et al and several other authors have also found the duration between GFS and $\mathrm{CE}$ to be a strong factor affecting the IOP control after $\mathrm{CE}$ and believe that filtering surgery needs sufficient time to develop adequately with the inflammation secondary to cataract surgery hampering this process. ${ }^{2,23,24}$ Relatively young age has been a wellknown risk factor for failure of GFS and is likely to be associated with excessive fibrosis during youth. ${ }^{21}$

Intraoperative complications occurred in two eyes $(2.6 \%)$ and intraoperative iris manipulation was required in five eyes $(6.5 \%)$ in the present study. The intraoperative iris manipulation was performed in small nondilating pupils in the form of pupilloplasty. However, we did not find an association between increased intraoperative iris manipulations or complications and 
qualified success. But intraoperative iris manipulation has been shown to be significantly associated with the need for additional medications or bleb needling.

In the present study, the probability of an eye requiring second glaucoma surgery was $0.03 \pm 0.97$. The probability that an eye would not require antiglaucoma medications beyond 2 months following CE was 0.96 . The bleb survival in our series was $92 \%$ at 6 months. Intraocular pressure and bleb function was maintained after CE with PCIOL implantation following a successful GFS with good visual recovery. We did not find any significant difference in the bleb appearance between pre- and postCE. In our series, patients with complete success had shorter follow-up than patients with qualified success (14 vs 35 months). We speculate that perhaps with extended follow-up the bleb function may deteriorate in the complete success group. Chen $e a^{2}$ observed a reduction in the size of the bleb after CE in $18 \%$ of their eyes but did not affect the survival rates. Some other authors have noted bleb scarring and shrinkage after $\mathrm{CE}_{\text {; }}^{5,13,16}$ occasionally with loss of IOP control. ${ }^{16}$

The limitations of the present study include its nonrandomized retrospective design, the variability in the timing of the CE following trabeculectomy and the arbitrary criteria for adding antiglaucoma medications following CE. Our results should be interpreted with caution because we defined success based on the level of IOP alone. Although we considered the severity of the disc damage and field loss in the management decisions regarding addition of antiglaucoma medications in the event of uncontrolled IOP following CE, we could not ensure consistency of such a modality because of the retrospective nature of our study.

In conclusion, CE following GFS resulted in continued IOP control and restoration of good acuity in $81.8 \%$ of eyes. Bleb function was not jeopardized by subsequent CE. In our study, patients aged $>60$ years undergoing CE within 5 months of trabeculectomy, using glaucoma medications before $\mathrm{CE}$ and demonstrating early postoperative IOP within 2 weeks of greater than $19 \mathrm{mmHg}$ were at significantly higher risk for loss of IOP control after CE.

\section{Acknowledgements}

This work was supported in part by the Hyderabad Eye Research Foundation, LV Prasad Eye Institute, Hyderabad, India.

\section{References}

1 Buratto L, Ferrari M. Extracapsular cataract surgery and intraocular lens implantation in glaucomatous eyes that had a filtering bleb operation. J Cataract Refract Surg 1990; 16: 315-319.
2 Chen PP, Weaver YK, Budenz DL, Feuer WJ, Parrish II RK. Trabeculectomy function after cataract extraction. Ophthalmology 1998; 105: 1928-1935.

3 Mills KB. Trabeculectomy: a retrospective long-term follow up of 444 cases. Br J Ophthalmol 1981; 65: 790-795.

4 Seah SKL, Jap A, Prata JA Jr, Baerveldt G, Lee PP, Heuer DK et al. Cataract surgery after trabeculectomy. Ophthalmic Surg Lasers 1996; 27: 587-594.

5 Dickens MA, Cashwell LF. Long-term effect of cataract extraction on the function of an established filtering bleb. Ophthalmic Surg Lasers 1996; 27: 9-14.

6 Wygnanski-Jaffe T, Barak A, Melamed S, Glovinsky Y. Intraocular pressure increments after cataract extraction in glaucomatous eyes with functioning filtering blebs. Ophthalmic Surg Lasers 1997; 28: 657-661.

7 Halikiopoulos D, Moster MR, Auzara-Blanco A, Wilson RP, Schmidt CM, Spaeth GL et al. The outcome of the functioning filter after subsequent cataract extraction. Ophthalmic Surg Lasers 2001; 32: 108-117.

8 Gunning FP, Greve EL. Intercapsular cataract extraction with implantation of the Galand disc lens: a retrospective analysis in patients with and without glaucoma. Ophthalmic Surg 1991; 22: 531-538.

9 Caprioli J, Park HJ, Weitzman M. Temporal corneal phacoemulsification combined with superior trabeculectomy: a controlled study. Trans Am Ophthalmol Soc 1996; 94: 451-463, discussion 463-468.

10 Manoj B, Chako D, Khan MY. Effect of extracapsular cataract extraction and phacoemulsification performed after trabeculectomy in intraocular pressure. J Cataract Surg 2000; 26: 75-78.

11 Burratto L, Ferrari M. Extracapsular cataract surgery and intraocular lens implantation in glaucomatous eyes that had a filtering bleb operation. J Cataract Refract Surg 1990; 16: 315-319.

12 Friedman DS, Jampel HD, Lubmoski LH, Kempen JH, Quigley H, Longdon $\mathrm{N}$ et al. Surgical strategies for coexisting glaucoma and cataract. An evidence-based update. Ophthalmology 2002; 109: 1902-1915.

13 Binkhorst CD, Huber C. Cataract extraction and intraocular lens implantation after fistulizing glaucoma surgery. $\mathrm{J} \mathrm{Am}$ Intraocular Implant Soc 1981; 7: 133-137.

14 Ostbaum SA. Glaucoma and intraocular lens implantation. J Cataract Refract Surg 1986; 12: 257-261.

15 Murchinson Jr JF, Shields MB. An evaluation of three surgical approaches for coexisting cataract and glaucoma. Ophthalmic Surg 1989; 20: 393-398.

16 Yamagami S, Araie M, Mori M, Mishima K. Posterior chamber intraocular lens implantation in filtered or nonfiltered glaucoma eyes. Jpn J Ophthalmol 1994; 38 : 71-79.

17 Antonios SR, Traverso CE, Tomey KF. Extracapsular cataract extraction using a temporal limbal approach after filtering operations. Arch Ophthalmol 1988; 106: 608-610.

18 Brooks AMV, Gillies WE. The effect of cataract extraction with implant in glaucomatous eyes. Aust NZ J Ophthalmol 1992; 20: 235-238.

19 Drolsum L, Haaskjold E. Extracapsular cataract extraction in eyes previously operated for glaucoma. Acta Ophthalmol (Copenh) 1994; 72: 273-278.

20 Krupin T, Fietl ME, Bishop KI. Postoperative intraocular pressure rise in open-angle glaucoma patients after cataract or combined cataract-filtration surgery. Ophthalmology 1989; 96: 579-584. 
21 Skuta GL, Parrish II RK. Wound healing in glaucoma filtering surgery. Surv Ophthalmol 1987; 32: 149-170.

22 Bigger JF, Becker B. Cataracts and primary open-angle glaucoma: the effect of uncomplicated cataract extraction on glaucoma control. Trans Am Acad Ophthalmol Otolaryngol 1971; 75: 260-272
23 Shields MB. Combined cataract extraction and guarded sclerectomy. Reevaluation in the extracapsular era. Ophthalmology 1986; 93: 366-370.

24 Shields MB. Textbook of Glaucoma, 4th ed. Williams and Wilkins: Baltimore, MD, 1998, p 566. 